Virtual Mentor. November 2003, Volume 5, Number 11.

doi: 10.1001/virtualmentor.2003.5.11.msoc1-0311

Medicine and Society

\title{
The Ethics of Quarantine
}

\section{Although civil rights questions come into play, quarantine as an attempt to control an infectious disease can still be used when certain conditions are met.}

\author{
Ross Upshur, MD, MSc, MA
}

Recent events, specifically the SARS epidemic and concerns for the use of infectious agents for bioterrorism, have brought public health practice into prominence as an integral aspect of health care. For example, in the SARS epidemic, public health authorities in Canada relied upon quarantine for the first time in several generations. The use of quarantine raises several ethical concerns. Many people believe that quarantine constitutes an unwarranted diminution of personal liberty, whereas others see it as an integral aspect of communicable disease control. The purpose of this article is to discuss some of the ethical issues raised by quarantine and present requirements for its justification from an ethical perspective. This discussion draws on recent scholarship on public health ethics, particularly with respect to autonomy-limiting actions by public health authorities.

There is no doubt that communicable diseases pose threats to populations, and the simple administration of health care is insufficient to control the spread of communicable diseases. Over the past century, public health has developed a series of strategies to apply at a population level to control the spread of communicable diseases. The mode of transmission for most communicable diseases is well known, and hence population-based strategies, such as contact tracing and isolation, are frequently used for situations such as TB. However, there are circumstances when communicable diseases threaten populations, and a broader public health strategy may be required. Quarantine is but one component of communicable disease control. On its own, it is unlikely to be effective, and it is by no means the sole method of controlling an outbreak.

There are 2 independent ethical considerations to consider here: whether the concept of quarantine is justified ethically and whether it is effective. It is also important to make a clear distinction between quarantine and isolation. Quarantine refers to the separation of those exposed individuals who are not yet symptomatic for a period of time (usually the known incubation period of the suspected pathogen) to determine whether they will develop symptoms. Quarantine achieves 2 goals. First, it stops the chain of transmission because it is less possible to infect others if one is not in social circulation. Second, it allows the individuals under surveillance to be identified and directed toward appropriate care if they become symptomatic. This is more important in diseases where there is presymptomatic shedding of virus. Isolation, on the other hand, is keeping those who have symptoms from circulation in general populations.

Justification of quarantine and quarantine laws stems from a general moral obligation to prevent harm to (infection of) others if this can be done [1]. Most democracies have public health laws that do permit quarantine. Even though quarantine is a curtailment of civil liberties, it can be broadly justified if several criteria can be met.

In my analysis, published in the Canadian Journal of Public Health, I identified 4 principles that must be met in order for public health to contemplate an autonomy-limiting strategy [2]. First, the harm principle must be met. In other words, there should be clear and measurable harm to others should a disease or exposure go unchecked. For quarantine, this infection should be spread from person to person. In diseases that are infectious but cannot be spread from person to person, such as anthrax, quarantine cannot be justified. 
Secondly, the proportionality, or least-restrictive-means, principle should be observed. This holds that public health authorities should use the least restrictive measures proportional to the goal of achieving disease control. This would indicate that quarantine be made voluntary before more restrictive means and sanctions such as mandatory orders or surveillance devices, home cameras, bracelets, or incarceration are contemplated. It is striking to note that in the Canadian SARS outbreak in the Greater Toronto area, approximately 30,000 persons were quarantined at some time. Toronto Public Health reports writing only 22 orders for mandatory detainment [3]. Even if the report is a tenfold underestimate, the remaining instances of voluntary quarantine constitute an impressive display of civic-mindedness.

Thirdly, reciprocity must be upheld. If society asks individuals to curtail their liberties for the good of others, society has a reciprocal obligation to assist them in the discharge of their obligations. That means providing individuals with adequate food and shelter and psychological support, accommodating them in their workplaces, and not discriminating against them. They should suffer no penalty on account of discharging their obligations to society.

Finally, there is the transparency principle. This holds that public health authorities have an obligation to communicate clearly the justification for their actions and allow for a process of appeal. If the above conditions can be met, there is a prima facie justification for the use of quarantine.

There are other frameworks for analysis of public health ethics. Nancy Kass [4] and James Childress [5], for example, have recently published frameworks for the ethical appraisal of public health programs. In their frameworks, the effectiveness of an intervention plays an important role in justifying public health intervention. This is a double-edged sword, however. In an emergency such as SARS, it would be desirable to have knowledge that your actions, including that of quarantine, would be effective. But being constrained from action due to lack of evidence of effectiveness would severely hamper public health response-and quite possibly lead to the further transmission of disease. As public health officers face these difficult dilemmas, it is important that they err on the side of public safety. It would be far better to defend oneself for unnecessary quarantine than to refrain from acting and expose individuals to a preventable disease, with subsequent morbidity and mortality. It should be noted that, despite controversies over quarantine, there is no clear or agreed-upon sense of what constitutes an effective quarantine.

This being said, it is important that there be a due process for quarantine. Barbera and colleagues have also addressed the issue of large-scale quarantine in the context of biological terrorism [6]. In their view, the effectiveness of quarantine is questionable and not justified on a mass basis. They indicate that quarantine actions have the capacity to cause harm. This is no doubt true. They do point out that there are several issues that need to be addressed, and they pose 3 major questions for a particular outbreak:

1. Do public health and medical analyses warrant the imposition of large-scale quarantine.

2. Are the implementation and maintenance of large-scale quarantine feasible?

3. Do the potential benefits of large-scale quarantine outweigh the possible adverse consequences?

Questions 1 and 2 are important and have been addressed in my framework. As to question 3, unfortunately, a priori, there may be very little information to work with. It is always hoped, of course, as a regulatory ideal, that more good than harm is done by intervention. I think that it is important for public health personnel to be mindful of this, particularly with respect to how people are supported. An effort should be made to minimize the long-term psychological impact and stigmatization of persons quarantined or otherwise affected.

In summary, then, quarantine is a blunt instrument to use in the control of infectious diseases. However, in some circumstances it is one of the only possible means of responding to an infectious disease threat. For example, early in the SARS outbreak in Toronto, when the disease showed rapid transmission to health care workers, the causative organism was unknown, as was the duration of communicability, mode of transmission, and incubation period. Many questions were unanswered. In this context of uncertainty, a prudent precautionary approach and the use of quarantine were likely justified. However, public health professionals must continually update their information in order to refine the exposure criteria, so that people are not needlessly quarantined. Hence, communication between public health professionals and clinicians is crucial. I also believe that physicians have a strong obligation to support public health in the control of communicable disease. Their actions in support of public health mandates are crucial in securing public credibility. Though many of these actions may be controversial, particularly when they begin to affect the livelihood of 
individuals, this is not an excuse for deviating from a control strategy. Transparency and communication are crucial in this regard.

\section{References}

1. Harris J, Holm S. Is there a moral obligation not to infect others? BMJ. 1995;311:1215-1217. View Article PubMed Google Scholar

2. Upshur RE. Principles for the justification of public health intervention. Can J Public Health. 2002;93:101-103. PubMed Google Scholar

3. Yaffe Barbara. SARS in Toronto: A Local Public Health Perspective. Presented at: SARS Symposium; September 17, 2003; University of Toronto.

4. Kass NE. An ethics framework for public health. Am J Public Health. 2001;91:1776-1782. View Article PubMed Google Scholar

5. Childress J, Faden R, Gaare R, et al. Public health ethics: mapping the terrain. J Law Med Ethics. 2001;30:170178.

View Article Google Scholar

6. Barbera J, Macintyre A, Gostin L, et al. Large-scale quarantine following biological terrorism in the United States: scientific examination, logistic and legal limits, and possible consequences. JAMA. 2001;286:2711-2717. View Article $\underline{\text { PubMed }}$ Google Scholar

Ross Upshur, MD, MA, MSc, is a research scholar and assistant professor in the Departments of Family and Community Medicine and Public Health Sciences and a member of the Joint Centre for Bioethics at the University of Toronto. He is currently the director of the Primary Care Research Unit at the Sunnybrook Campus of the Sunnybrook and Women's College Health Sciences Centre.

virtualmentor@ama-assn.org

The viewpoints expressed on this site are those of the authors and do not necessarily reflect the views and policies of the AMA.

(C) 2003 American Medical Association. All Rights Reserved. 